http://jmscr.igmpublication.org/home/ ISSN (e)-2347-176x ISSN (p) 2455-0450

crossref DOI: https://dx.doi.org/10.18535/jmscr/v7i10.58

Journal Of Medical Science And Clinical Research

\title{
MRCP as a Challenging Tool in Evaluation of Pancreatico-Biliary Pathologies
}

\author{
Dr Kuncham Nagabalaji ${ }^{1}$, Dr Pagadala Padmavathi ${ }^{2}$, Dr Julius N Toppo ${ }^{3}$ \\ ${ }^{1}$ Final Year Post Graduate, Department of Radiodiagnosis KIMS \& RF \\ ${ }^{2}$ Second Year Postgraduate, Department of Radiodiagnosis, KIMS \& RF \\ ${ }^{3}$ Professor, Department of Radiodiagnosis, KIMS \& RF
}

\begin{abstract}
Background: Biliary system imaging can be done invasively (Ultrasonography, Computed Tomography) or noninvasively (Endoscopic Retrograde Cholangio Pancreatography). Currently we opt for non invasive Magnetic Resonance Cholangio-Pancreatography [MRCP] to detect biliary pathologies accurately.

MRCP is noninvasive with inherent high contrast resolution, multi planar acquisition capability and with avoidance of contrast and ionizing radiation; is able to create projectional type images. It can also reveal extraductal tumours and is unaffected by bowel gas giving high resolution images with better sensitivity.
\end{abstract}

Aim:

1. To evaluate patients with clinical suspicion of pancreato - biliary ductal pathologies with conventional Magnetic Resonance Imaging (MRI) and Magnetic Resonance CholangioPancreatography (MRCP).

2. To compare the diagnostic accuracy of MRCP with Ultrasonography (USG) for the evaluation of pancreato - biliary ductal diseases.

Materials and Methods: The study comprised of a total no of hundred cases suffering from various pancreato - biliary ductal diseases of all age groups and either sex in the previous 18 months who were referred to Radiology department, KIMS, Amalapuram.

Results: In our study, we found that USG has $95.45 \%$ accuracy in diagnosing benign and malignant pancreato-biliary pathologies while MRCP is $97 \%$ accurate when compared to the standard histopathology.

Conclusion: Based on our study, we conclude that MRI serves as an accurate and non-invasive, nonionizing imaging method for evaluation of biliary anatomy and pathology; and can be considered as the gold standard for evaluating pancreato-biliary pathologies compared to USG.

Keywords: MRI, Magnetic Resonance Cholangio-Pancreatography (MRCP), ultrasonography (USG), pancreato-biliary pathologies. 


\section{Introduction}

Pancreato-biliary ductal system is a common site for a wide variety of pathologies causing obstructive jaundice which necessitates the investigation for the site of obstruction and for the etiology of causative pathologies; as it helps in planning the appropriate treatment strategy by the clinicians. To investigate such a variety of pathologies, imaging often requires a multimodality approach.

In current scenario, Ultrasonography (USG) serves as an initial screening modality of choice in suspected cases of pancreato-biliary ductal diseases which is complimented by Computed Tomography (CT). However these modalities have a limited sensitivity in detecting calculi within common bile duct (CBD) especially in the distal portion as it is obscured by bowel gas, food bolus within the duodenum or by adipose tissue. CT also has a limited role in detecting biliary calculi and strictures. It is said that CT has only $90 \%$ sensitivity in detecting biliary calculi ${ }^{(1)}$.

These cases require invasive procedures like IntraVenous Cholangiography (IVC); Endoscopic Retrograde Cholangio-Pancreatography (ERCP) and Percutaneous Transhepatic Cholangiography (PTC). IVC has a limited role as it causes incomplete opacification of the biliary system in $30-40 \%$ of cases $^{(2,3)}$ ERCP and PTC require biliary intervention and use of contrast media; with approximately 1-4\% sepsis rate ${ }^{(4)}$.

The most challenging part faced by the radiologists in pancreato-biliary ductal imaging lies in the accurate diagnosis of neoplasms (like cholangiocarcinoma) and inflammatory conditions (like primary sclerosing cholangitis). These tumours either have a primary origin from the ducts themselves or have a secondary metastatic spread to pancreato - biliary tree from tumours of liver, gall bladder, pancreas or adjacent lymph nodes. Therefore cause and level of obstruction is mandatory before establishing precise therapy. ${ }^{(7)}$ In this scenario, Magnetic Resonance CholangioPancreaticography (MRCP) emerged as a relatively new diagnostic modality and has revolutionized the imaging of biliary and pancreatic ducts. It serves as a dynamic, accurate, non-invasive, non-sedative, operator independent, radiation free tool which is unaffected by bowel gas giving high resolution images without contrast usage in evaluating pancreato - biliary ductal diseases. $^{(8)}$

Itprecisely identifies the nature of the disease (infection, tumour, calculus \& others); with localization and extent of involvement suggesting the type of pathology. It helps to guide the biopsy and drainage procedures, suggest the method of therapy (medical and/or surgical), and help in assessing the response to therapy.

In this present study we have prospectively studied 100cases by MRI with MRCP and USG who were suffering from various diseases of biliary tract and/or pancreas and tried to evaluate the efficacy of MRI with MRCP as an imaging modality of choice.

\section{Aims and Objectives}

1. To evaluate patients with clinical suspicion of pancreato - biliary ductal pathologies with conventional Magnetic Resonance Imaging (MRI) and Magnetic Resonance Cholangio-Pancreatography (MRCP).

2. To compare the diagnostic accuracy of MRCP with Ultrasonography (USG) for the evaluation of pancreato - biliary ductal diseases.

3. To prove that the MRCP is one of the best imaging modality for evaluation of pancreato - biliary ductal diseases.

4. To assess the pitfalls in MRCP in evaluating the pancreato - biliary ductal diseases.

\section{Methodology}

Study Design: Hospital based prospective study.

Source of Data and Sample Size: A total no of hundred cases suffering from various pancreato biliary ductal diseases of all age groups and either sex were included in this study. 
Study Period: Eighteen months; from 2016 to 2018.

Study Area: Department of Radiodiagnosis, Konaseema Institute of Medical Sciences and Research Foundation (KIMS \& RF), Amalapuram.

Statistical Analysis: Data will be analyzed by sensitivity, specificity, positive predictive value and negative predictive value.

\section{Method of Collecting Data}

The study protocol was approved by the ethical committee.

Informed consent was obtained from all the cases with suspected various pancreato - biliary ductal diseases; in a period of 18 months from 2016 to 2018 who were subjected for the study.

A structured pre-prepared case proforma was used to enter the patient details, detailed clinical history, past and family history of any significant medical problems; and physical examination to meet our inclusion criteria.

All the cases had undergone ultrasonography and most them had been diagnosed on USG prior to MRI-MRCP examination.

Inclusion Criteria: All cases referred for MRCP to the Department of Radiodiagnosis, KIMS \& RF, Amalapuram with suspected pancreato biliary ductal diseases in prior ultrasonography, over a period of 18 months.

Exclusion Criteria: We have excluded:

- Pregnant women.

- Patients who underwent recent interventional procedures.

- Post-operative cases.

- Patients with cardiac pacemakers / ferromagnetic aneurismal clips / metallic orthopaedic implants / cochlear implants / intraorbital metallic foreign bodies.

- Severe claustrophobia.

- Subjects unwilling to participate in the study.

\section{Preparation for USG:}

- All the patients were instructed to come with empty stomach on the day of procedure.

Equipment used: PHILIPS Clearvue 650 machine; both curvilinear and linear probes were used for the study.

\section{Patient preparation for MRI with MRCP:}

- All the patients were instructed to fast for 6 hours prior to examination.

- All the metallic belongings removed prior to the examination.

- All images were obtained in supine position with breath holding and parameters were individualized to optimize each for a suspended breath hold of about $15 \mathrm{~s}$.

- In few uncooperative and critically ill patients, respiratory triggering was used.

Equipment used: PHILIPS Achieva 1.5 T MRI machine, Philips CLEARVUE 650 Ultrasonography machine.

\section{Results and Observations}

Our study was conducted to determine the MRI MRCP in the evaluation of patients with obstructive jaundice Vs USG. A total of 100 cases who were clinically diagnosed with pancreato biliary ductal diseases; and who underwent USG and MR-MRCP were included in the present study.

In our study, we observed that the most common cause among various pancreato - biliary ductal diseases is of benign etiology; and males are commonly affected in both benign (47 cases) and malignant (11 cases) diseases. 
Table No. 02: Sex - Wise Distribution of Cases Showing Various benign Pancreato - Biliary Ductal diseases as Observed on USG and MRI - MRCP.

\begin{tabular}{|c|c|c|c|c|c|c|}
\hline \multirow[b]{2}{*}{ TYPE OF DISEASE } & \multicolumn{3}{|c|}{ AS OBSERVED ON USG } & \multicolumn{3}{|c|}{$\begin{array}{c}\text { AS OBSERVED ON MRI- } \\
\text { MRCP }\end{array}$} \\
\hline & $\mathbf{M}$ & $\mathbf{F}$ & TOTAL & $\mathbf{M}$ & $\mathbf{F}$ & TOTAL \\
\hline CHOLELITHIASIS (ISOLATED) & $\mathbf{0 7}$ & 11 & 18 & 07 & 11 & 18 \\
\hline CHOLEDOCHAL CYST & $\mathbf{0 2}$ & 05 & $\mathbf{0 7}$ & $\mathbf{0 3}$ & 09 & 12 \\
\hline CALCULOUS CHOLECYSTITIS & 05 & $\mathbf{0 2}$ & $\mathbf{0 7}$ & 06 & $\mathbf{0 3}$ & 09 \\
\hline $\begin{array}{l}\text { ACALCULOUS } \\
\text { CHOLECYSTITIS }\end{array}$ & $\mathbf{0 3}$ & 01 & 04 & 04 & 01 & 05 \\
\hline $\begin{array}{l}\text { CHOLEDOCHOLITHIASIS } \\
\text { (ISOLATED) }\end{array}$ & 01 & 02 & 03 & $\mathbf{0 3}$ & 05 & 08 \\
\hline $\begin{array}{l}\text { CHOLEDOCHOLITHIASIS } \\
\text { WITH CHOLELITHIASIS }\end{array}$ & $\mathbf{0 2}$ & $\mathbf{0 2}$ & 04 & 04 & 05 & 09 \\
\hline $\begin{array}{l}\text { PANCREATITIS } \text { (ACUTE } / \\
\text { CHRONIC / ACUTE ON } \\
\text { CHRONIC) }\end{array}$ & 07 & 02 & 09 & 10 & 02 & 12 \\
\hline HEPATIC ABSCESS & 01 & - & 01 & 01 & - & 01 \\
\hline GB POLYP & 02 & - & $\mathbf{0 2}$ & $\mathbf{0 2}$ & - & 02 \\
\hline CHOLANGITIS & 01 & - & 01 & $\mathbf{0 2}$ & - & $\mathbf{0 2}$ \\
\hline BENIGN BILIARY STRICTURES & $\mathbf{0 3}$ & $\mathbf{0 1}$ & 04 & 05 & $\mathbf{0 2}$ & $\mathbf{0 7}$ \\
\hline TOTAL & 34 & 26 & 60 & 47 & 38 & 85 \\
\hline
\end{tabular}

In our study, we observed that the most common benign cause of pancreato - biliary ductal diseases as observed on MRI - MRCP was cholelithiasis; which was either isolated $(21.18 \%$ of all benign cases) or associated with choledocholithiasis $(10.59 \%$ of all benign cases) or cholecystitis (10.59\% of all benign cases); males are commonly affected.

Table No. 03: Sex Wise Distribution of Cases Showing various malignant Pancreato - Biliary Ductal Diseases as Observed on USG and MRI - MRCP.

\begin{tabular}{|l|c|c|c|c|c|c|}
\hline \multirow{2}{*}{ TYPE OF DISEASE } & \multicolumn{3}{|c|}{ AS OBSERVED ON USG } & \multicolumn{3}{c|}{ AS OBSERVED ON MRI - } \\
\cline { 2 - 7 } & $\mathrm{M}$ & $\mathrm{F}$ & TOTAL & M & F & TOTAL \\
\hline GALL BLADDERCARCINOMA & 01 & 01 & 02 & 02 & 02 & 04 \\
\hline CHOLANGIOCARCINOMA & - & 01 & 01 & 02 & 01 & 03 \\
\hline KLATSKIN TUMOUR & - & - & - & 01 & - & 01 \\
\hline $\begin{array}{l}\text { PERIAMPULLARY } \\
\text { CARCINOMA }\end{array}$ & - & - & - & 02 & - & 02 \\
\hline PANCREATIC CARCINOMA & 02 & - & 02 & 03 & 01 & 04 \\
\hline $\begin{array}{l}\text { HEPATOCELLULAR } \\
\text { CARCINOMA }\end{array}$ & 01 & - & 01 & 01 & - & 01 \\
\hline TOTAL & 04 & 02 & 06 & 11 & 04 & 15 \\
\hline
\end{tabular}

In our study of various pancreato - biliary ductal diseases, as observed on MRI - MRCP, gall bladder carcinoma and pancreatic carcinoma (each of $26.67 \%$ respectively, among all malignant causes) were found to be the most common malignant causes; all of these causes have male predominance. 
Table No. 04: Histopathological / Surgical Correlation of Various Benign Pancreato - Biliary Ductal Diseases Diagnosed on USG and MRI - MRCP.

\begin{tabular}{|c|c|c|c|}
\hline TYPE OF DISEASE & USG & MRI - MRCP & HPE / SURGERY \\
\hline CHOLELITHIASIS (ISOLATED) & 18 & 18 & 18 \\
\hline CHOLEDOCHAL CYST & 07 & 12 & $\begin{array}{c}12 \text { (SURGERY / FOLLOW } \\
\text { UP) }\end{array}$ \\
\hline CALCULOUS CHOLECYSTITIS & 07 & 09 & $08 *$ \\
\hline ACALCULOUS CHOLECYSTITIS & 04 & 05 & $04 *$ \\
\hline CHOLEDOCHOLITHIASIS & 03 & 08 & 08 \\
\hline $\begin{array}{l}\text { CHOLEDOCHOLITHIASIS WITH } \\
\text { CHOLELITHIASIS }\end{array}$ & 04 & 09 & 09 \\
\hline $\begin{array}{lllr}\text { PANCREATITIS } & \text { (ACUTE } & \text { / } \\
\text { CHRONIC } & / & \text { ACUTE } & \text { ON } \\
\text { CHRONIC) } & & & \\
\end{array}$ & 09 & 12 & $11^{\#}$ \\
\hline HEPATIC ABSCESS & 01 & 01 & 01 \\
\hline GB POLYP & $\mathbf{0 2}$ & $\mathbf{0 2}$ & $\mathbf{0 2}$ \\
\hline CHOLANGITIS & 01 & 02 & $02($ FOLLOW - UP) \\
\hline BENIGN BILIARY STRICTURES & 04 & 07 & 07 \\
\hline TOTAL & 60 & 85 & 82 \\
\hline
\end{tabular}

On histopathological correlation, we observed that the most common benign cause of pancreato biliary ductal diseases on USG and MRI - MRCP was cholelithiasis; which was either isolated (21.95\% of all benign cases) or associated with choledocholithiasis (10.98\% of all benign cases) or cholecystitis $(9.76 \%$ of all benign cases $)$.
*MRI - MRCP has diagnosed 14 cases of cholecystitis (calculous and acalculous), out of which 12 cases were diagnosed as chlecystitis on HPE. The remaining 2 turned out to be gall bladder carcinoma.

\# Out of 12 pancreatitis cases diagnosed on MRI MRCP, one case turned out to be pancreatic carcinoma on HPE.

Table No. 05: Histopathological / Surgical Correlation of Various Malignant Pancreato - Biliary Ductal Diseases Diagnosed On USG and MRI - MRCP.

\begin{tabular}{|l|c|c|c|}
\hline TYPE OF DISEASE & USG & MRI -MRCP & HPE / SURGICAL \\
\hline GALL BLADDER CARCINOMA & 02 & 04 & $06 *$ \\
\hline CHOLANGIOCARCINOMA & 01 & 03 & 03 \\
\hline KLATSKIN TUMOUR & - & 01 & 01 \\
\hline PERIAMPULLARY CARCINOMA & - & 02 & $\mathbf{0 3}^{\#}$ \\
\hline PANCREATIC CARCINOMA & $\mathbf{0 2}$ & $\mathbf{0 4}$ & $\mathbf{0 4}$ \\
\hline HEPATOCELLULAR CARCINOMA & $\mathbf{0 1}$ & $\mathbf{0 1}$ & $\mathbf{0 1}$ \\
\hline TOTAL & $\mathbf{0 6}$ & $\mathbf{1 5}$ & $\mathbf{1 8}$ \\
\hline
\end{tabular}

On histopathological correlation, we observed that the most common malignant cause of pancreato biliary ductal diseases on USG and MRI - MRCP was gall bladder carcinoma $(33.33 \%$ of all malignant causes).

*Out of the total 6 cases of gall bladder carcinoma which were diagnosed on histopathology, only 4 were detected on MRI - MRCP. The remaining 2 were misdiagnosed as cholecystitis.
\# Out of 3 cases of histopathologically periampullary carcinoma, only 2 cases were diagnosed accurately on MRI - MRCP. The remaining one case was diagnosed as pancreatic carcinoma on MRI - MRCP.

Inconclusive USG findings were excluded for the calculation of sensitivity, specificity, PPV, NPV. 
Table No. 07: Role of MRI - MRCP vs HPE / Surgery in Evaluating Benign and Malignant Causes of Pancreato - Biliary Ductal Diseases

\begin{tabular}{|l|c|c|c|}
\hline & \multicolumn{2}{|c|}{ HPE / SURGERY } & \\
\hline MRI - MRCP & BENIGN & MALIGNANT & TOTAL \\
\hline BENIGN & $\mathbf{8 2}$ & $\mathbf{0 3}$ & $\mathbf{8 5}$ \\
\hline MALIGNANT & -- & 15 & 15 \\
\hline TOTAL & $\mathbf{8 2}$ & 18 & 100 \\
\hline
\end{tabular}

Chart No. 04: Role of MRI - MRCP vs HPE / Surgery in Evaluating Benign and Malignant Causes of Pancreato - Biliary Ductal Diseases.

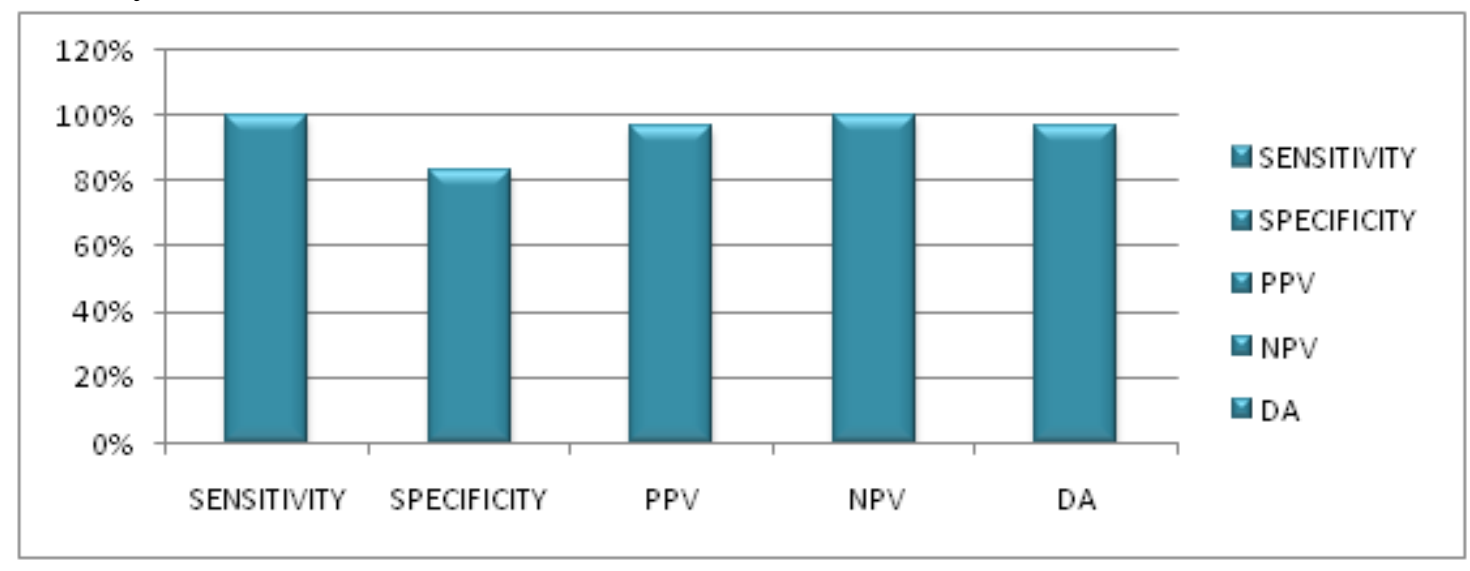

Chart 05: Comparison of Diagnostic Values between USG and MRI - MRCP in Causing Pancreato Biliary Ductal Diseases

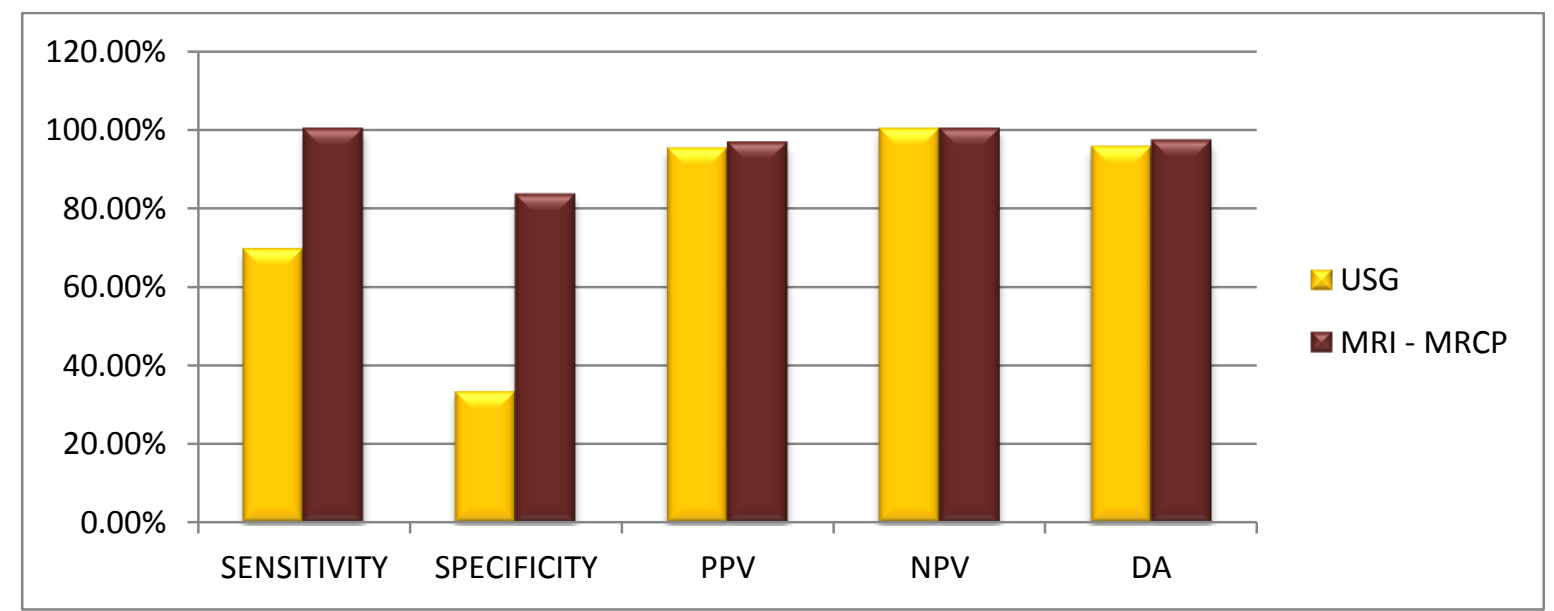

From the above chart, it can be inferred that for diagnosing the cause of pancreato - biliary ductal diseases, MRI with MRCP has sensitivity (100\%) and specificity (83.33\%) far greater than USG. 
JMSCR Vol||07||Issue||10||Page 340-353||October

Malignant Distal CBD Stricture

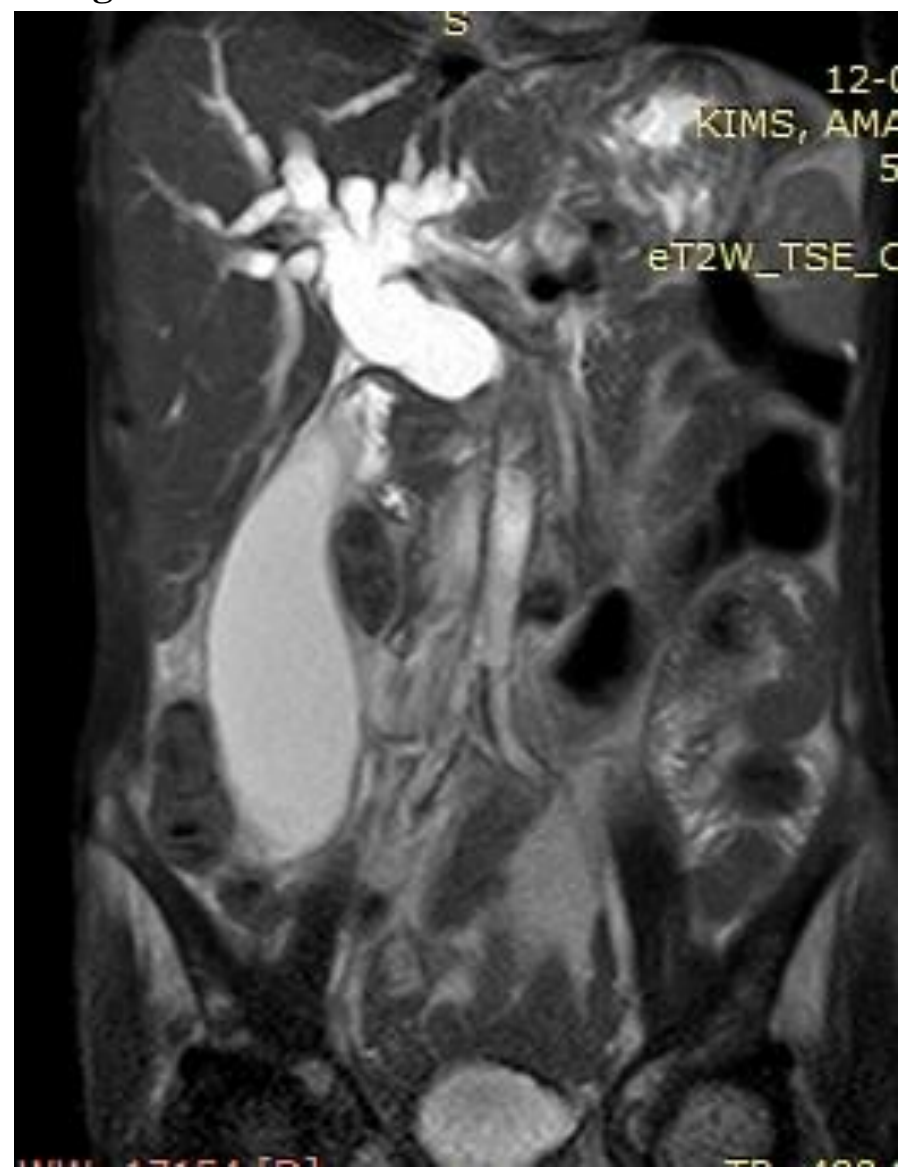

T2W TSE coronal image showing significantly dilated CBD with abrupt cut off at distal end.

\section{Cholangiocarcinoma}

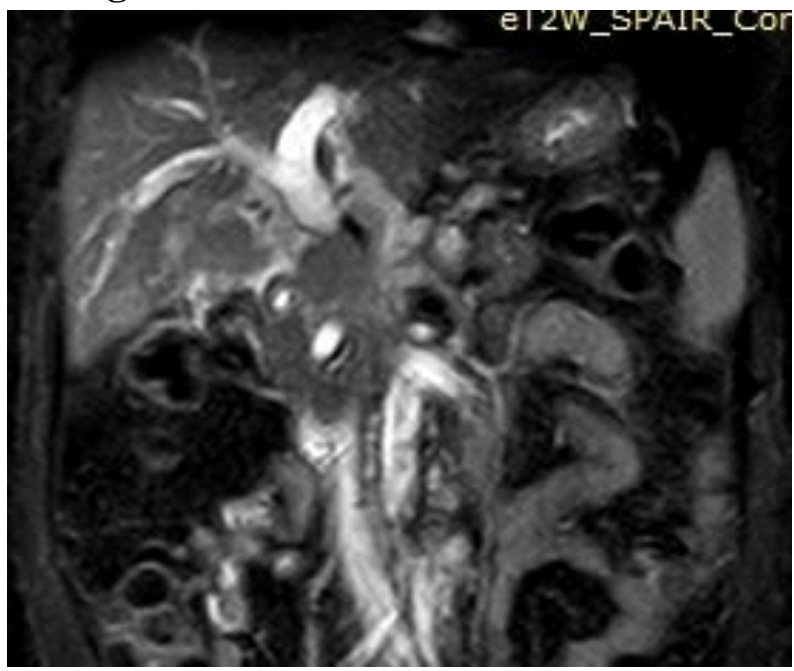

T2W SPAIR coronal image shows hypointense lobulated mass lesion in distal CBD with extension into gallbladder.

\section{Carcinoma Gall Bladder}

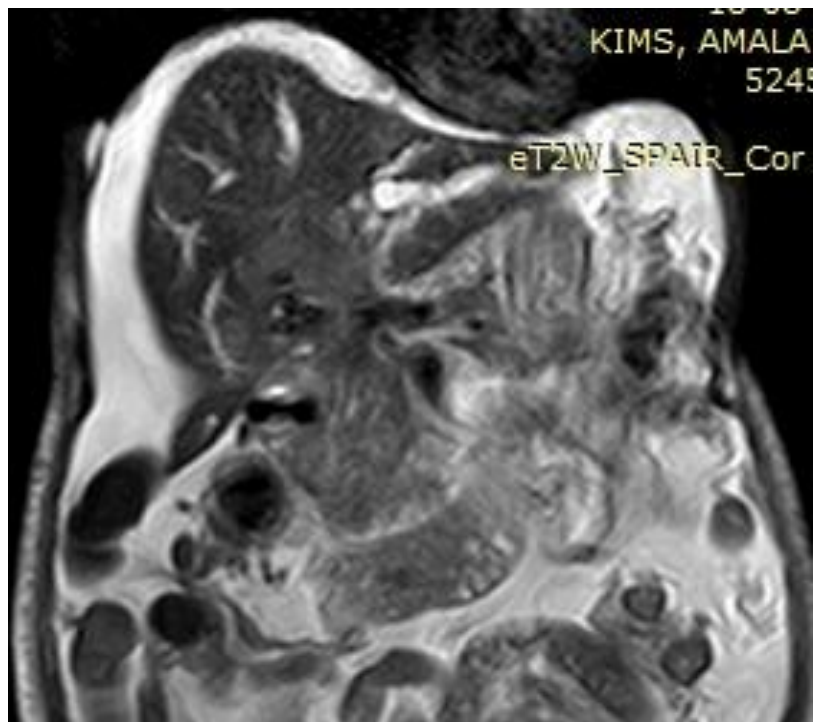

T2WE SPAIR coronal image shows heterogenous mass involving gall bladder with infiltration into the adjacent liver parenchyma.

\section{Periampullary Carcinoma}

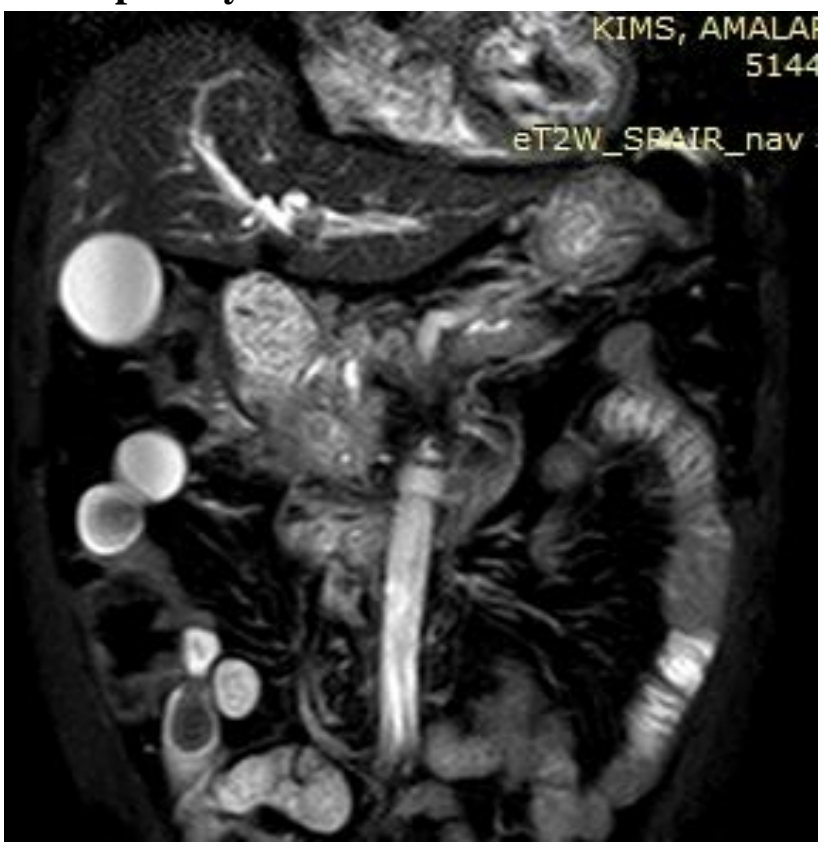




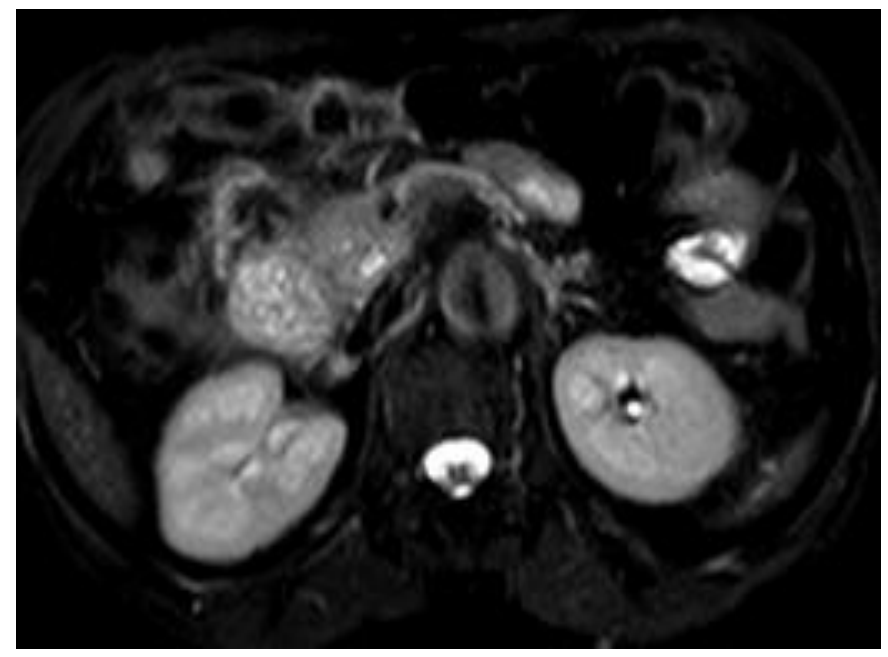

T2W SPAIR coronal and axial images show heterogenous predominantly hyperintense mass lesion at periampullary region.

\section{Case 6: Hepatic Abscess}

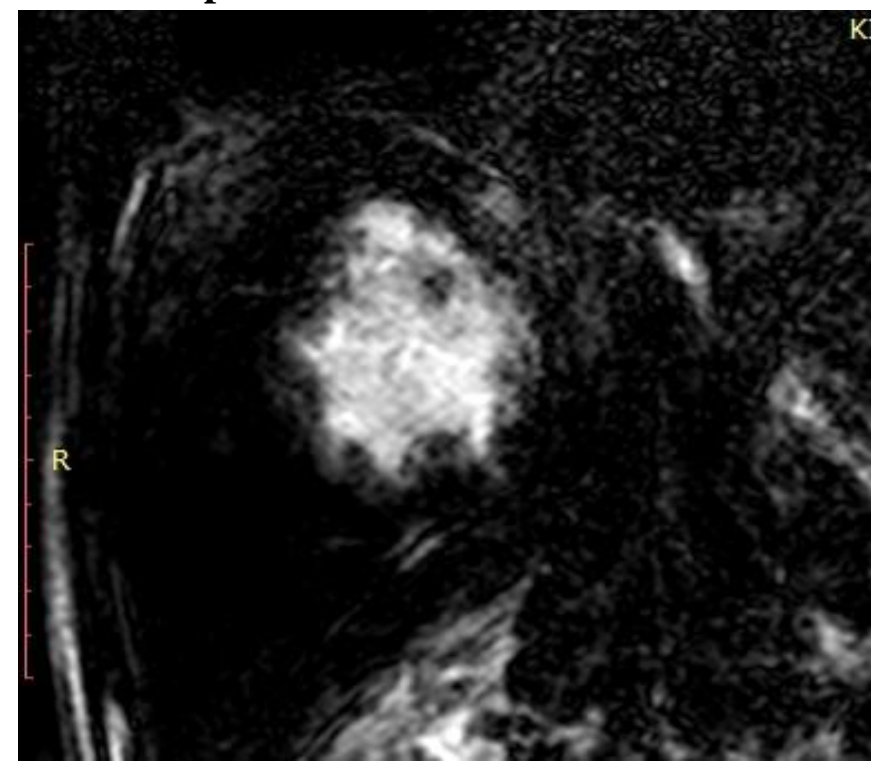

Heterogeneously hyperintense mass lesion within the liver with thickened wall.

\section{Discussion}

Diagnosing patients with suspected biliary or pancreatic pathologies in their early stage is most important for patient care and management. Knowledge of the advantages and disadvantages of each technique are needed to determine the appropriate work up of patients with these pathologies.

In our study, we have studied 100 cases suffering from various causes of pancreato-biliary ductal diseases. All these cases underwent USG prior to MRI - MRCP.
Incidence of various pancreato - biliary ductal diseases:

Among various benign pancreato - biliary ductal diseases, cholelithiasis is the most common pathology observed which either is an isolated pathology $(21.95 \%)$ or is associated with choledocholithiasis or cholecystitis in $9.76 \%$ in either of these conditions; followed by pancreatitis (acute / chronic / acute or chronic) in $13.41 \%$ and benign biliary strictures in $8.54 \%$.

Among various malignant pancreato - biliary ductal diseases, gall bladder carcinoma (33.33\%) is the most common pathology.

\section{Age Distribution}

The youngest patient was 3 years old presented with choledochal cyst and oldest patient was of 80 years old with gall bladder carcinoma. Maximum numbers of patients $(51 \%)$ were adults in the age group of 31-60years with a male preponderance of $58 \%$; and with a male: female ratio of $1.38: 1$.

\section{Clinical Features}

Almost all of our cases presented with jaundice and abdominal pain. Few cases of choledochal cysts were detected incidentally. Most common sign encountered in our study was icterus. This is comparable to the study by Nirhale et al where icterus was the most common presenting sign accounting for $21.67 \%$. $^{(9)}$

Characterization of benign pancreato - biliary ductal diseases

\section{Cholelithiasis}

This was the most common pathology observed among all the pancreato - biliary ductal diseases; which was either isolated $(21.95 \%$ of all benign cases) or associated with choledocholithiasis (10.98\% of all benign cases) or cholecystitis ( $9.76 \%$ of all benign cases).

USG was able to detect gall bladder calculi in all of the cases with $100 \%$ accuracy. Few cases (4 out of 18 isolated cholelithiasis cases) showed a single large calculus at the neck region; thereby obstructing the bile flow.

However in one case there is suspicion for microlithiasis on USG, where MRCP was quite 
helpful in confirming the diagnosis which was emphasized by Norero et al too. ${ }^{(10)}$

One case has features of adenomyomatosis on ultrasonography which showed the classical hyperechoic wall with multipletiny calcifications giving it a comet tail appearance. MRCP showed classical "pearl necklace sign" with multiple filling defects arranged in a curvilinear fashion within the lumen of gall bladder.MRI has offered diagnostic accuracy in differentiating adenomyomatosis from gall bladder carcinoma. Yoshimitsu et al ${ }^{(88)}$ stated that MRI provides $93 \%$ diagnostic accuracy in differentiating adenomyomatosis from carcinoma gall bladder.

\section{Choledocholithiaisis}

In imaging of benign lesions ( $\mathrm{n}=82), \mathrm{CBD}$ calculi were found in a total of 17 cases, out of which 9 cases showed associated cholelithiasis; in which gall stones are seen to migrate into the biliary ductal system causing secondary dilatation of common bile duct. MRI with MRCP diagnosed CBD with GB calculi in all 9 cases showing $100 \%$ accuracy; while USG could detect only 4 cases when associated with GB calculi. USG showed difficulty in picking up distal CBD calculus in 10 patients due to obscuration by bowel gas or adipose tissue.

These are diagnosed clearly by MRI with $100 \%$ accuracy; where calculus appeared as an area of signal void. This shows that MR with MRCP is superior to USG in detecting CBD calculi and other distal CBD pathologies. Cholelithiasis and choledocholithiasis are best visualized on $\mathrm{T} 2 \mathrm{~W}$ images as they appear as filling defects within the bright signal background of bile.

Study done by Romagnuolo et al ${ }^{(11)}$ stated that MRCP is highly sensitive for choledocholithiasis; specially in detecting ductal calculi $<3 \mathrm{~mm}$ calibre, with an overall pooled sensitivity of $95 \%$ in one meta-analysis of patients with suspected biliary disease.

In spite of its effectiveness in detecting choledocholithiasis, MRCP cannot accurately depict any alteration in adjacent organs, only being restricted to biliary tree. As Gautier emphasized, MRCP seems to be an efficient screening method for patients at risk for a future ERCP. ${ }^{(12)}$

\section{Benign biliary strictures}

Benign biliary stricture was diagnosed in 7 patients. MRI with MRCP clearly showed benign nature of stricture in all 7 cases approaching 100\% accuracy. MRCP showed clearly the length of the stricture segment very well and differentiated stricture as malignant and benign. Our study is in concordance with Bhatt et al; In their study they found $100 \%$ accuracy forMRCP in diagnosing benign CBD strictures. ${ }^{(13)}$

\section{Choledochal cysts}

Choledochal cysts were diagnosed in 12 cases on MRI with MRCP; with a diagnostic accuracy of $100 \%$. In all these cases, MRI - MRCP yielded diagnostic information by providing exact anatomic map in pre surgical evaluation. Our study is in concordance with Bhatt et al; in their study they found $100 \%$ accuracy for MRCP in diagnosing anatomical variants. ${ }^{(13)}$

Out of the 12 cases, majority (8) were of type I (Ic -5 cases, Ib -2 cases, Ia -1 case) category in Todani classification while the rest were categorized under IV (IVa - .3 cases, IVb - 1 case).

ERCP serves as the most definitive and reliable diagnostic method; but requires general anaesthesia in children and is contraindicated in acute pancreatitis and cholangitis.

\section{Cholecystitis}

The next common pathology was found to be cholecystitis (14 cases); out of which the majority (9) were associated with gallstones. This proves that cholelithiasis is a strong predisposing factor for cholecystitis. USG clearly demonstrates gall bladder wall thickening with subtle pericholecysticfluid in 11 out of 14 cases. MRI MRCP detected all 14 cases. Hence it is further helpful in demonstrating the accuracy of this pathology. This is in concordance with the study done by Hakannson et al in 2000; which stated that MRCP is far more superior than USG in 
detecting cholecystitis as MRCP is more sensitive to pericholecystic collection. ${ }^{(14)}$

\section{Gall bladder polyp}

Two cases of gall bladder polyp were studied; where USG accurately diagnosed both the cases as they appeared as subcentimetric echogenic foci adherent to the wall of gall bladder with no evidence of posterior acoustic shadow. On MRI, the polyp appeared as T2 hyperintense foci adherent to the wall of gall bladder. The diagnosis was confirmed on histopathology.

\section{Cholangitis}

Two cases of acute cholangitis were reported out of which one was identified on USG which accurately demonstrated the cause of the obstruction (gallstones); and the level of the obstruction. MRI - MRCP on the other hand had almost $100 \%$ accuracy in demonstrating acute cholangitis. MRI - MRCP demonstrated the classical increased periductal signal intensity on $\mathrm{T} 2 \mathrm{~W}$ along with demonstration of cause and level of obstruction.

\section{Pancreatitis}

Pancreatitis was observed in a total of 11 cases; out of which majority (6) was acute pancreatitis, one case was of acute on chronic type while the rest (4) were of chronic pancreatitis. The majority were males (10). Most of them had significant alcohol abuse history. The second most common predisposing factor is gall stones. USG has accurately diagnosed 9 out of these 11 cases, in which 6 were of acute pancreatitis while 3 were of chronic pancreatitis. The pancreas appeared bulky and hypoechoic with peripancreatic fluid collections in few. Two cases demonstrated pseudocyst formation.

On MRI - MRCP, 12 cases were diagnosed as pancreatitis out of which one case of pancreatic carcinoma was misdiagnosed as mass forming chronic pancreatitis; as DWI was not included in the regular protocol. In the rest, MRCP accurately delineated the changes within the pancreas with maximum sensitivity to changes in the parenchymal intensity due to oedema.
MRCP is far more superior to USG in detecting peripancreatic collections.

One case has demonstrated walled - off necrosis in chronic necrotizing pancreatitis on MRI MRCP which on T2 weight images show collections and debris within the pancreas with dilated pancreatic duct. However the air within the collection is not well appreciated on MRI. Hence MRI with MRCP was more sensitive in detecting walled - off pancreatic necrosis (WOPN). Comparison study done by Rana SS et al in 2015 demonstrated that MRI is more sensitive in detecting necrotic debris in WOPN. ${ }^{(15)}$

\section{Hepatic abscess}

A single case of hepatic abscess was demonstrated; which appeared as a hypoechoic lesion with low level internal echoes on USG while on MRI, the central portion of the lesion appears cystic, and the "rind" exhibits variable intensities on T1- and T2-weighted imaging. The diagnosis was confirmed by FNAC.

\section{Characterization of malignantpancreato -} biliary ductal diseases

\section{Gall bladder carcinoma}

In imaging of malignant lesions $(\mathrm{n}=18)$, we observed six cases of gall bladder carcinoma; out of which 2 cases were diagnosed accurately on USG which was used as an initial investigative tool. These cases showed nodular wall thickening on USG. However USG could not accurately delineate the extent of spread of tumour and stage the disease. Hence USG can be used as a primary investigative tool but cannot be used for staging carcinoma gall bladder. These findings are comparable to the study of sonographic evaluation of gall bladder carcinoma done by Bach et al in 1998. ${ }^{(16)}$

Out of the 6 cases with gall bladder carcinoma MRI with MRCP diagnosed 4 cases with $100 \%$ accuracy. Among these, two patients had liver metastases shown clearly by both the modalities. In such cases, MRI can be used as an investigative tool and the staging will be very accurate. MRI detected all 2 cases and is helpful in detecting subtle lesions in liver and local spread 
and helped in pre-surgical staging. Study done by Kaur et al in 2018 to evaluate MRCP over ultrasonography in various biliary obstruction cases stated that MRCP is more sensitive and accurate than USG. ${ }^{(17)}$

One case presented with extension into peritoneal fat and head of the pancreas. Majority (3) of the cases had significant bile duct dilatation secondary to gallbladder carcinoma which was easily characterized on MRI - MRCP. However in 2 cases MRI - MRCP could not differentiate benign from malignant as they showed subtle focal wall thickening with small intramural nodule. Out of these, one case was misdiagnosed as xanthogranulomatous cholecystitis on MRI - MRCP as DWI and contrast imaging were not performed. However histopathology proved the diagnosis to be gall bladder carcinoma.

\section{Cholangiocarcinoma}

In 3 patients with cholangiocarcinoma MRI with MRCP diagnosed all 3 cases with 100\% accuracy with the help of conventional MRI, while USG showed suspicion where a heterogenous mass with intermediate echogenicity noted compressing the liver. MRI with MRCP offered $100 \%$ diagnostic accuracy which is far more superior to USG. When studying correlation between imaging findings and histopathological diagnosis we found a stricture with malignant characteristics at MRCP to be the most predictive sign of malignancy. Our results were in accordance with the study done by Singh et al in 2014; ${ }^{(18)}$ which demonstrated that USG is $66.67 \%$ sensitive and $100 \%$ specific while MRI with MRCP is $83.33 \%$ sensitive (far more than USG) and $100 \%$ specific in detecting cholangiocarcinoma.

\section{Klatskin tumour}

One case was diagnosed to have Klatskin tumour on MRI - MRCP which demonstrated a malignant stricture with shouldering and abrupt cut-off at the bifurcation of common hepatic duct. MRCP was useful in visualizing the exact biliary tree map and also helpful in staging the disease; which is essential in planning and choosing the appropriate management. The role of MRI - MRCP in staging and planning treatment of hilar cholangiocarcinoma was emphasized by Masselli et al in 2007. ${ }^{(19)}$

\section{Periampullary carcinoma}

Periampullary growth was found in a total of 2 cases on histopathology out of which one case was diagnosed accurately on MRI with MRCP; which showed irregular thickening of ampullary margin with significant upstream dilatation of CBD and pancreatic duct giving it a classical "double duct" sign which helped more in arriving imaging diagnosis. However in the other case, presence of double duct sign was not seen as the mass is not completely obstructing the pancreatic duct. In this case, MRI - MRCP has diagnosed it to be a pancreatic carcinoma; but turned out to be a periampullary carcinoma on histopathology. Study done by Kim et al ${ }^{(20)}$ in 2008 has demonstrated that double duct sign is visible in only $52 \%$ cases approximately. On T2 weighted coronal images, hypointense, bi-lobed pancreatic mass with compressive mass effect upon the descending duodenum and abrupt termination of the dilated CBD was noted giving it an "Inverted figure 3" sign; which was first described by Frostberg in 1938. ${ }^{(21)}$ One patient was diagnosed to have stricture among the periampullary growth patients on MRI - MRCP, due to technical fault and due to patient non-cooperation.

\section{Pancreatic carcinoma}

A total of 4 cases of pancreatic carcinoma were diagnosed on histopathology. Out of which two cases were diagnosed accurately on USG which was initially performed as a screening tool in patients presenting with jaundice and abdominal pain to rule out choledocholithiasis and to look for biliary dilatation. In our study the accuracy of USG was approximately 50\% in diagnosing pancreatic carcinoma; which was comparable to the study done by Rickes et al in 2002 stating that the accuracy of USG was around $50-70 \%$ in diagnosing pancreatic carcinoma. ${ }^{(22)}$

One case showed suspicious malignancy where conventional $\mathrm{T} 1$ weighted imaging along with 
MRCP is beneficial in detecting the associated lymphadenopathy and hepatic metastases.

\section{Hepatocellular carcinoma}

A single case of hepatocellular carcinoma was studied, which was diagnosed accurately on USG which appeared as a hypoechoic mass with areas of fatty change and necrosis in a background of cirrhotic liver and with intrahepatic biliary dilatation. However USG could not detect the extent of spread and is operator dependent. Hence the accuracy is limited; which is similar to the comparative study done by Jalli et al in 2015 which demonstrated the limited accuracy of USG (78.12\%) while incorporation of MRI along with DWI has greatly increased the accuracy to $94.79 \%$. (23)

The patient underwent MRI - MRCP to evaluate biliary tumour thrombi. This case showed filling defects in the right hepatic and common hepatic ducts with intrahepatic bile duct dilatation. Conventional MRI images showed T1 hypointense and $\mathrm{T} 2$ hyperintense intrabiliary tumour thrombus which is quite a rare finding.

\section{Limitations and Pit Falls of the Present Study}

* Since ours is a tertiary care referral hospital in remote location most patients coming to our hospital are of low socioeconomic group with financial constraints for routine MRCP in all patients.

* Number of malignant cases in this study sample is very low.

* Since contrast MRI is not incorporated in routine MRCP protocol evaluation of lesions other than biliary tree and pancreatic malignancies is limited.

* Breathing artefacts in non-cooperative patients cause significant image deterioration which increases imaging time to yield diagnostic images.

\section{Recommendations}

* We recommend routine use of MRCP in all cholelithiasis and choledocholithiasis cases to evaluate various anomalies related to biliary confluence and cystic duct insertion especially when laparocopic surgery is contemplated.

* We recommend routine acquisition of T1, T2 weighted images along with 3D volumetry MRI images in all cases to look for ancillary findings which alter course of management and in all cases of pancreatitis.

* We recommend routine screening USG in all cases before MRCP especially in alcoholics and obese patients to look for portal hypertension which helps in deciding the type of anaesthesia to be administered.

* We recommend incorporation of dynamic post contrast MRI imaging in all cases of suspected malignancy.

* We recommend incorporation of Diffusion weighted imaging (DWI) in cases with hepatic lesions on USG.

Based upon our study, the following conclusions can be drawn:

- $\quad \mathrm{MRI}-\mathrm{MRCP}$ is a non-invasive, nonionizing imaging technique which provides accurate diagnosis in evaluating pancreato-biliary system.

- However the initial screening modality of choice is still Ultrasonography.

- It is useful in children, critically ill and obese patients with ease.

- It is superior to USG in showing the distal CBD as well as pancreatic duct.

- The inherent multi-planar capability of MRI with MRCP makes it superior to other modalities in characterizing the lesion.

\section{References}

1. Dawson $\mathrm{P}$, Adam A, Benjamin I S .Intravenous Cholangiography revisited. Clinical Radiology 1993; 47:223-225.

2. Patel JC, Mc-Innes GC, Bagley JS, Needham G, Krukowski ZH. The role of intravenous cholangiography in preoperative assessment for laparoscopic 
cholecystectomy. The British journal of radiology. 1993 Dec;66(792):1125-7.

3. Wallner BK, Schumacher KA, Weidenmaier W, Friedrich JM. Dilated biliary tract: evaluation with MR cholangiography with a T2-weighted contrast-enhanced fast sequence. Radiology. 1991 Dec;181(3):805-8..

4. Schwartz LH, Coakley FV, Sun Y, Blumgart LH, Fong Y, Panicek DM. Neoplastic pancreaticobiliary duct obstruction: evaluation with breath-hold MR cholangiopancreatography. AJR. American journal of roentgenology. 1998 Jun;170(6):1491-5

5. Soto JA, Barish MA, Yucel EK, Siegenberg D, Ferrucci JT, Chuttani R. Magnetic resonance cholangiography: comparison with endoscopic retrograde cholangiopan-creatography.

Gastroenterology. 1996 Feb 1;110(2):58997.

6. Larena JA, Astigarraga E, Saralegui I, Merino A, Capelastegui A, Calvo MM. Magnetic resonance cholangiopancreatography in the evaluation of pancreatic duct pathology. The British journal of radiology. 1998 Oct;71(850):1100-4.

7. James E Pearls .Advanced MRI - From Head to Toe. 2002; 3:211-246.

8. Takehara Y. Fast MR imaging for evaluating the pancreaticobiliary system. European journal of radiology. 1999 Mar 1;29(3):211-32.

9. Nirhale DS, Kulkarni GC, Shingade P, Chavan S, Sonawane T, Dhende M. Role of magnetic resonance cholangiopancreatography in diagnosing pancreatobiliary pathologies: a prospective study. International Surgery Journal. 2018 May 24;5(6):2233-7.

10. Norero E, Norero B, Huete A, Pimentel F, Cruz F, Ibanez L, Martinez J, Jarufe N. Accuracy of magnetic resonance cholangiopancreatography for the diagnosis of common bile duct stones. Revistamedica de Chile. 2008 May;136(5):600-5.

11. Romagnuolo J, Bardou M, Rahme E, Joseph L, Reinhold C, Barkun AN. Magnetic resonance cholangiopancreatography: a meta-analysis of test performance in suspected biliary disease. Annals of internal medicine. 2003 Oct 7;139(7):547-57.

12. Gautier G, Pilleul F, Crombe-Ternamian A, Gruner L, Ponchon T, Barth X, Valette PJ. Contribution of magnetic resonance cholangiopancreatography to the management of patients with suspected common bile duct stones. Gastroentérologie Clinique et biologique. 2004 Feb 1;28(2):129-34.

13. Bhatt C, Shah PS, Prajapati HJ, Modi J. Comparison of diagnostic accuracy between USG and MRCP in biliary and pancreatic pathology. Indian Journal of Radiology and Imaging. 2005 May 1;15(2):177.

14. Håkansson $\mathrm{K}$, Leander $\mathrm{P}$, Ekberg $\mathrm{O}$, Håkansson HO. MR imaging in clinically suspected acute cholecystitis: a comparison with ultrasonography. Actaradiologica. 2000 Jul;41(4):322-8.

15. Rana SS, Chaudhary V, Sharma R, Sharma V, Chhabra P, Bhasin DK. Comparison of abdominal ultrasound, endoscopic ultrasound and magnetic resonance imaging in detection of necrotic debris in walled-off pancreatic necrosis. Gastroenterology report. 2015 Jan 7;4(1):50-3.

16. Bach AM, Loring LA, Hann LE, Illescas FF, Fong Y, Blumgart LH. Gallbladder cancer: can ultrasonography evaluate extent of disease?.Journal of ultrasound in medicine. 1998 May;17(5):303-9.

17. Kaur A., Malaviya A, Deepika, Kaur N. K. Comprehensive evaluation of MRCP versus ultra sonography in biliary 
obstruction. Int J Med Res Rev 2018;6

(03):143-152. doi:10.17511/ijmrr. 2018.i03.03.

18. Singh A, Mann HS, Thukral CL, Singh NR. Diagnostic accuracy of MRCP as compared to ultrasound/CT in patients with obstructive jaundice. Journal of clinical and diagnostic research: JCDR. 2014 Mar;8(3):103.

19. Masselli G, Gualdi G. Hilarcholangiocarcinoma: MRI/MRCP in staging and treatment planning. Abdominal imaging. 2008 Jul 1;33(4):444-51.

20. Kim TU, Kim S, Lee JW, Woo SK, Lee TH, Choo KS, Kim CW, Kim GH, Kang DH. Ampulla of Vater: comprehensive anatomy, MR imaging of pathologic conditions, and correlation with endoscopy. European journal of radiology. 2008 Apr 1;66(1):48-64

21. Frostberg N. A characteristic duodenal deformity in cases of different kinds of peri-vaterial enlargement of the pancreas. Actaradiologica. 1938 Mar(2):164-73.

22. Rickes S, Unkrodt K, Neye H, Ocran KW, Wermke W. Differentiation of pancreatic tumours by conventional ultrasound, unenhanced and echo-enhanced power Doppler sonography. Scandinavian journal of gastroenterology. 2002 Jan 1;37(11): 1313-20.

23. Jalli R, Jafari SH, Sefidbakht S, Kazemi K. Comparison of the accuracy of DWI and ultrasonography in screening hepatocellular carcinoma in patients with chronic liver disease. Iranian Journal of Radiology. 2015 Jan;12(1). 